Full-length article

\title{
Assessment of the inhibition potential of Licochalcone A against human UDP-glucuronosyltransferases
}

Hong Xin ${ }^{\text {a }}$, Xiao-Yi Qi ${ }^{\text {bcc }}$, Jing-Jing Wu ${ }^{\text {b }}$, Xin-Xin Wang ${ }^{\mathrm{b}}$, Yan $\mathrm{Li}^{\mathrm{b}}$, James Y. Hong ${ }^{\mathrm{d}}$, Wei $\mathrm{He}^{\mathrm{a}}$, Wei $\mathrm{Xu}^{\mathrm{a},{ }^{*}}$, Guang-Bo Ge ${ }^{\mathrm{b},{ }^{*}}$, Ling Yang ${ }^{\mathrm{b}}$

${ }^{a}$ First Affiliated Hospital of Harbin Medical University, Harbin 150001, China.

${ }^{\mathrm{b}}$ Laboratory of Pharmaceutical Resource Discovery, Dalian Institute of Chemical Physics, Chinese Academy of Sciences, Dalian 116023, China.

${ }^{c}$ First Affiliated Hospital of Dalian Medical University, Dalian 116024, China.

${ }^{\mathrm{d}}$ Department of Pharmacy Practice, College of Pharmacy, University of Illinois at Chicago, USA.

\section{Correspondence}

Tel: +8613796059990

E-mail address: 13796059990@163.com

$\&$

Tel: +8641184676961

E-mail address: geguangbo@dicp.ac.cn 


\section{Abstract}

Licochalcone A (LCA) is a major bioactive compound in Licorice, a widely used herbal medicine. In this study, the inhibitory effects of LCA against human UDP-glucuronosyltransferases (UGTs) and LCA associated herb-drug interactions were systematically investigated. Our results demonstrated that LCA displayed broad-spectrum inhibition against human UGTs. LCA exhibited strong inhibitory effects against UGT1A1, 1A3, 1A4, 1A6, 1A7, 1A9, and 2B7 (both $\mathrm{IC}_{50}$ and $K_{i}$ values lower than $5 \mu \mathrm{M}$ ), while showing moderate inhibitory effects against UGT1A8, 1A10, 2B4, 2B15, and 2B17. The inhibitory effects of LCA against two major UGTs, including UGT1A1 and 1A9, were further investigated in human liver microsomes (HLMs), where the potential risks of LCA via inhibition of UGT1A1 and 1A9 were predicted by combining the in vitro inhibitory data and physiological data. The results from this study also showed that several LCA-containing products were able to increase the area under the curve (AUC) of the substrates that were predominantly metabolized by UGT1A1 or 1A9. These findings together demonstrate that LCA has a potent and broad-spectrum inhibitory effect against most human UGTs and thus suggest that much caution should be exercised when high-dose LCA is co-administered with UGT substrates.

Keywords: Licochalcone A (LCA), UDP-glucuronosyltransferases (UGT), Broad-spectrum inhibitor, Herb-drug interactions (HDIs) 


\section{Introduction}

Licorice is roots of Glycyrrhiza inflate (also named Gan-Cao in China), which has been widely used in herbal medicine and as food traditionally in Asia. Licorice is one of the major ingredients used for preparing several famous Traditional Chinese Medicine (TCM) products, such as as Mistura Glycyrrhizae Composita, HuoxiangZhengqi Tincture, and Naolejing Oral Liquid (Pharmacopoeia of the People`s Republic of China, 2005). Licochalcone A (Fig. 1) is a major bioactive flavonoid isolated from the roots of licorice (Hatano et al., 1988). In the past thirty years, a wide variety of pharmacological studies have shown that LCA possesses many potential biological activities, including anti-parasitic (Ziegler et al., 2004), anti-inflammatory (Kolbe et al., 2006), anti-oxidative (Haraguchi et al., 1998), anti-bacterial (Friis-Møller et al., 2002), and anti-tumor activities (Yo et al., 2009; Xiao et al., 2011; Park et al., 2015). In China, licorice is listed in Chinese Pharmacopoeia and the List of Herbal Materials that Can Be Used for Health Foods under the Law on Food Hygiene by State Food and Drug Administration, and its daily dosage can be as high as up to $60 \mathrm{~g} /$ day (Liu et al., 2014). As the most abundant constituent in commercially available licorice in China, the concentration of LCA ranges in $4-10 \mathrm{mg} / \mathrm{g}$ of dried licorice $(0.4-1 \% \mathrm{w} / \mathrm{w})$, which translates into upwards of 240-600 mg of LCA as the daily dose (Zhang and Ye, 2009; Shibata, 2000). In such cases of high daily intake of licorice, much attention should be paid to monitor the potential herb-drug interactions (HDIs) since LCA-containing herbal preparations are commonly used with many therapeutic drugs in China.

In recent years, many studies have demonstrated that crude extracts of licorice and LCA can inhibit the activities of human cytochrome P450 enzymes (CYPs) and P-glycoprotein 
transporters (P-gps) (Choi et al., 2014; Qiao et al., 2014; Choi et al., 2013). More recently, we have also found that LCA exhibits broad-spectrum inhibitory effects toward human CYP enzymes including CYP1A2, 2C8, 2C9, 2C19, and 3A4 (He et al., 2015). These studies suggest that LCA can reduce the elimination of many therapeutic drugs that are substrates of CYPs and P-gp, which may cause subsequent HDIs. It is also possible that HDIs can result from herbal-associated inhibition against major phase II metabolizing enzymes ( $\mathrm{Na}$ et al., 2011; Choi et al., 2011; Li et al., 2012; Zheng et al., 2014; Wang et al., 2015 ). Unlike the extensive investigations on LCA interactions with CYPs, there is currently no reported evidence on the inhibitory effects of LCA and other major constituents of licorice against common phase II metabolizing enzymes. Thus, it is important to evaluate the potential inhibitory effects of LCA or other major constituents isolate from licorice against human phase II metabolizing enzymes, such as UDP-glucuronosyltransferases (UGTs).

UDP-glucuronosyltransferases are superfamily of membrane-bound enzymes that are responsible for glucuronidation of many endogenous molecules (e.g., bilirubin, steroid hormones, thyroid hormones, bile acids, and fat-soluble vitamins) and exogenous compounds (e.g., drugs, chemical carcinogens, and environmental toxins) (Ritter, 2000). The formation of glucuronides is one of the most important detoxification pathways among vertebrates (Jancova et al., 2010). Moreover, a large number of different UGTs that exhibit a wide range of substrates are distributed throughout human body, which makes them a particularly important group of phase II metabolizing enzymes (Kiang et al., 2005). To date, at least twenty-two human UGTs have been identified, and they are classified into three subfamilies, namely UGT1A, UGT2A, and UGT2B, based on their amino acid sequence (Rowland et al., 
2013). Most UGTs are expressed in the liver, but some isoforms, such as UGT1A7, UGT1A8, and UGT1A10, are expressed predominantly in the gastrointestinal tract (Gregory et al., 2004). Human intestinal UGTs are considered as important determinants in intestinal metabolism, especially for those oral phenolic compounds, such as flavonoids and courmarins (Zhang et al., 2007; Wu et al., 2011; Xia et al., 2015). Therefore, the inhibition against human UGTs in the gastrointestinal tract may make a great impact on the oral bioavailability of many phenolic drugs or natural products.

This study aimed to investigate the inhibitory effects of LCA against human UGTs. To this end, twelve commercially available human UGTs were used to screen the inhibitory potential of LCA in vitro, while trifluoperazine (TFP) N-glucuronidation (for UGT1A4) and 4-methylumbelliferone (4-MU) O-glucuronidation (for all other UGT isoforms) were used as probe reactions. The dose-dependent inhibition curve and the half maximal inhibitory concentration $\left(\mathrm{IC}_{50}\right)$ of LCA against each UGT isoform were determined firstly. For those UGT isoforms which could be potently inhibited by LCA $\left(\mathrm{IC}_{50}<10 \mu \mathrm{M}\right)$, the inhibition mechanism and the inhibition constant $\left(K_{i}\right)$ values of LCA against human UGTs were carefully characterized. Moreover, the inhibitory effects of LCA against UGT1A1 and UGT1A9 were further investigated in human liver microsomes considering their key roles in glucuronidation of both endogenous compounds and therapeutic drugs, as well as the potent inhibitory effects of LCA against these two enzymes. Finally, the changes in the AUCs of UGT1A1 and UGT1A9 substrates following co-administration of LCA-containing herbal products were predicted based on a series of available data on literature. 


\section{Materials and Methods}

\subsection{Chemicals and reagents}

Licochalcone A (>98\%) was purchased from Sichuan Victory Biotechnology Co., Ltd (Sichuan, China). 4-Methylumbelliferone (4-MU), 4-methylumbelliferone glucuronide (4-MUG), trifluoperazine, propofol, propofol O-glucuronide and uridine-5-diphosphoglucuronic acid (UDPGA) were purchased from Sigma-Aldrich (St. Louis, MO, USA). NCHN (N-3-carboxypropyl-4-hydroxy-1,8-naphthalimide) and NCHN O-glucuronide were synthesized by the authors, the structures of NCHN and O-glucuronide were fully characterized as described previously (Lv et al., 2015). The purities of NCHN and O-glucuronide were higher than $98 \%$ by LC-UV. Pooled human liver microsomes from twenty-five donors were purchased from Research Institute for Liver Diseases (Shanghai,

China). Twelve recombinant human UGT Supersomes ${ }^{\mathrm{TM}}$ (UGT1A1, 1A3, 1A6, 1A7, 1A8, 1A9, A10, 2B4, 2B7, 2B15, and 2B17) expressed in baculovirus-infected insect cells were purchased from BD Gentest (Woburn, MA, USA) and stored at $-80{ }^{\circ} \mathrm{C}$ until use. Tris- $\mathrm{HCl}$ buffer ( $\mathrm{pH}$ 7.4) was prepared by using Millipore filtered water (Millipore, Bedford, USA) and stored at $4{ }^{\circ} \mathrm{C}$ until use. All other reagents were of the highest purity commercially available or HPLC grade.

\subsection{Assessment of inhibitory effects of LCA against human UGTs}

Unlike CYP inhibition assays, in which many specific probe substrates can be used and human liver microsomes are generally utilized as enzyme source, only a limited number of specific substrates can be employed in the evaluation of UGT inhibition (Liu et al., 2012). Therefore, recombinant UGT isoforms are commonly used as enzyme source when 
evaluating the inhibitory potential of LCA against UGTs. 4-Methylumbelliferone (4-MU), a non-selective substrate for UGTs, is typically used as a probe substrate for all UGTs isoforms except for UGT1A4 (Uchaipichat et al., 2004).

In this study, 4-MU was incubated with recombinant UGT1A1, 1A3, 1A6, 1A7, 1A8, 1A9, 1A10, 2B4, 2B7, 2B15, and 2B17 in both absence and presence of varying LCA concentrations $(1 \mu \mathrm{M}, 10 \mu \mathrm{M}$, and $100 \mu \mathrm{M})$. The positive controls used were nilotinib (0-20 $\mu \mathrm{M})$ for UGT1A1, magnolol $(0-10 \mu \mathrm{M})$ for UGT1A9, and androsterone $(1-100 \mu \mathrm{M})$ for UGT2B7 (Ai et al., 2014; Zhu et al., 2012; Uchaipichat et al., 2006). Each positive control compound was dissolved in dimethyl sulfoxide (DMSO) and its final concentration was $1 \%$ (v/v). Corresponding to the reported values of $K_{m}$ or $S_{50}$ for each UGT isoform, the concentrations of 4-MU were as follows: $110 \mu \mathrm{M}$ for UGT1A1, $1200 \mu \mathrm{M}$ for UGT1A3, 110 $\mu \mathrm{M}$ for UGT1A6, $30 \mu \mathrm{M}$ for UGT1A7, $750 \mu \mathrm{M}$ for UGT1A8, $30 \mu \mathrm{M}$ for UGT1A9, $30 \mu \mathrm{M}$ for UGT1A10, $1000 \mu \mathrm{M}$ for UGT2B4, $350 \mu \mathrm{M}$ for UGT2B7, $250 \mu \mathrm{M}$ for UGT2B15, and $2000 \mu \mathrm{M}$ for UGT2B17 (Uchaipichat et al., 2004). The incubation time and protein concentrations were carried out according to the previous studies with slight modifications (Zhu et al., 2012; Dong et al., 2012). In brief, a typical incubation mixture (a total volume of $200 \mu \mathrm{L}$ ) contained recombinant UGT isoforms (final concentration: $0.125 \mathrm{mg} / \mathrm{ml}, 0.05 \mathrm{mg} / \mathrm{ml}$, $0.025 \mathrm{mg} / \mathrm{ml}, 0.05 \mathrm{mg} / \mathrm{ml}, 0.025 \mathrm{mg} / \mathrm{ml}, 0.05 \mathrm{mg} / \mathrm{ml}, 0.05 \mathrm{mg} / \mathrm{ml}, 0.25 \mathrm{mg} / \mathrm{ml}, 0.05 \mathrm{mg} / \mathrm{ml}$, $0.2 \mathrm{mg} / \mathrm{ml}$, and $0.5 \mathrm{mg} / \mathrm{ml}$ for UGT1A1, 1A3, 1A6, 1A7, 1A8, 1A9, 1A10, 2B4, 2B7, 2B15, and 2B17, respectively), $50 \mathrm{mM}$ Tris- $\mathrm{HCl}$ buffer ( $\mathrm{pH} 7.4), 5 \mathrm{mM} \mathrm{MgCl}_{2}$, and $4 \mathrm{mM}$ UDPGA. After pre-incubation at $37^{\circ} \mathrm{C}$ for $5 \mathrm{~min}$, the reaction was initiated by the addition of UDPGA. The incubation time was $60 \mathrm{~min}$ for UGT1A1, 1A3, 1A10, 2B4, 2B7, 2B15, and 2B17, and 
$30 \mathrm{~min}$ for $1 \mathrm{~A} 6,1 \mathrm{~A} 7,1 \mathrm{~A} 8$, and $1 \mathrm{~A} 9$. The reactions were terminated by adding $200 \mu \mathrm{L}$ ice-cold acetonitrile (equal volume to incubation mixture) followed by centrifugation at 20,000 $\times \mathrm{g}$ for $10 \mathrm{~min}$ by using Allegra 64R centrifuge (Beckman, USA). The aliquots of supernatant were then taken for ultra-fast liquid chromatography-ultraviolet spectrometry (UFLC-UV) analysis.

Trifluoperazine (TFP) is a specific probe substrate for UGT1A4 that is used in this study. TFP $\left(50 \mu \mathrm{M}\right.$, near its $K_{\mathrm{m}}$ value $)$ was incubated with recombinant UGT1A4 $(0.1 \mathrm{mg} / \mathrm{ml})$ at $37^{\circ} \mathrm{C}$ for $30 \mathrm{~min}$ in both absence and presence of varying LCA concentrations $(1 \mu \mathrm{M}, 10 \mu \mathrm{M}$, and $100 \mu \mathrm{M})$ (Guo et al., 2013; Song et al., 2015). Then the samples were treated and determined as described above.

\subsection{Kinetic analyses for LCA-associated inhibition on recombinant human UGTs}

Half maximal inhibitory concentration $\left(\mathrm{IC}_{50}\right)$ was determined using various concentrations of LCA. $\mathrm{IC}_{50}$ values were calculated by non-linear regression analysis using Graphpad Prism 6.0 (San Diego, CA, USA). Inhibition kinetic parameters $\left(K_{i}\right)$ were determined by using various concentrations of 4-MU/TFP in the presence or absence of varying concentrations of LCA. The reversible inhibitory type can be categorized into competitive inhibition, non-competitive inhibition, or mixed inhibition (Lu et al., 2013). The corresponding reaction rates were determined by utilizing various concentrations of 4-MU/TFP and LCA. The inhibition kinetic type was evaluated through determining the intersection point in the Dixon and Lineweaver-Burk plots. The second plot of slopes from Lineweaver-Burk plot versus LCA concentrations was utilized to calculate the corresponding inhibition parameter $\left(K_{i}\right)$ value. 
2.4 Kinetic analyses for LCA against NCHN O-glucuronidation in both HLM and UGT1A1

$\mathrm{N}$-(3-carboxy propyl)-4-hydroxy-1,8-naphthalimide (NCHN) was a newly developed fluorescent probe for human UGT1A1 (Lv et al., 2015). In this study, NCHN is used as a selective probe substrate for UGT1A1 to determine the inhibitory effects of LCA against NCHN O-glucuronidation in both HLM and UGT1A1 (Wang et al., 2015). The IC $_{50}$ values were determined in two different enzyme sources by using various LCA concentrations, while $K_{i}$ values were determined by using various concentrations of NCHN in the presence or absence of different concentrations of LCA. In brief, NCHN ( $40 \mu \mathrm{M}$, final concentration) was incubated with $\mathrm{HLM}(0.2 \mathrm{mg} / \mathrm{ml})$ at $37^{\circ} \mathrm{C}$ for $30 \mathrm{~min}$, or incubated with UGT1A1 $(0.06$ $\mathrm{mg} / \mathrm{ml})$ at $37^{\circ} \mathrm{C}$ for $90 \mathrm{~min}$, in the presence of different LCA concentrations $(0-40 \mu \mathrm{M})$. The reactions were terminated and treated as described above, and the aliquots of supernatant were taken for liquid chromatography-fluorescence detector analysis.

\subsection{Kinetic analyses for LCA against propofol O-glucuronidation in both HLM and UGT1A9}

Propofol $O$-glucuronidation has been widely used as a probe reaction to phenotype UGT1A9 activity in human liver (Zhu et al., 2012; Miners et al., 2011). To explore whether LCA displayed substrate-dependent inhibition behavior against UGT1A9, as well as to determine the inhibitory effects of LCA against UGT1A9 in HLM, propofol was used as a selective probe substrate for UGT1A9 in this study (Rowland et al., 2008). Briefly, to get the $\mathrm{IC}_{50}$ and the $K_{i}$ values of LCA against propofol $O$-glucuronidation, propofol was incubated with HLM or UGT1A9 in presence of varying LCA concentrations $(0-20 \mu \mathrm{M})$, while all incubations were performed at $37^{\circ} \mathrm{C}$ for $20 \mathrm{~min}$, with the final protein concentrations were $0.05 \mathrm{mg} / \mathrm{ml}$ for HLM and UGT1A9. The reactions were quenched by adding $200 \mu \mathrm{L}$ 
acetonitrile with 4-MUG $(1.5 \mu \mathrm{M})$ as internal standard. The samples were treated as described above, and the aliquots of supernatant were taken for LC-MS/MS analysis.

\subsection{Prediction of the DDI potential in vivo}

The magnitudes of inhibitory interactions of LCA against UGT1A1 and UGT1A9 were estimated as the ratio of the area under the plasma concentration-time curve (AUC) in the presence and absence of the inhibitor. The ratio was predicted using the following equation (Kirby and Unadkat., 2010).

$$
\text { AUC ratio }=\frac{1}{f_{\text {hep }} \cdot\left(\frac{1 / E_{\mathrm{h}}}{\left(1 / E_{h}-1\right) \cdot\left(1+I / K_{i}\right)+1}\right)+\left(1-f_{\text {hep }}\right)}
$$

Where $f_{\text {hep }}$ is the percentage of hepatic clearance mediated by UGT1A1 or UGT1A9; $E_{\mathrm{h}}$ is the hepatic extraction ratio; $I$ is the maxmum concentration of inhibitor in human plama, and $K_{i}$ $(\mu \mathrm{M})$ is the inhibitory constant.

\subsection{Instruments and analytical conditions}

The LC system (Shimadzu, Kyoto, Japan) contained a SCL-30A system controller, two LC-30AD pumps, a SIL-30AC auto sampler, a DGU-20AC vacuum degasser, a CTO-30AC column oven, a SPD-20AVP UV detector and a RF-20A fluorescence detector. An ODS (5 $\mu$, 150.0 mm×2.1 mm, Acchrom Technology Corp., Beijing, China) analytical column was used and the column temperature was kept at $40{ }^{\circ} \mathrm{C}$. Acetonitrile (A) and Millipore filtered water with $0.2 \%$ formic acid (B) were used as the mobile phase at a flow rate of $0.4 \mathrm{ml} / \mathrm{min}$. For samples containing 4-MU and its glucuronide, the following gradient condition was used: 0-2.0 $\min , 90 \% \mathrm{~B} ; 2.01-9.0 \min 90 \% \mathrm{~B}-25 \% \mathrm{~B} ; 9.01-10.0 \min 25 \% \mathrm{~B}-5 \% \mathrm{~B} ; 10.01-13.0$ min, $5 \% \mathrm{~B} ; 13.01-16.0 \mathrm{~min}$, balance to $90 \% \mathrm{~B}$. The detection wavelength was set at $317 \mathrm{~nm}$. 
For the detection of TFP and its glucuronide, the following gradient condition was used: 0-9.0 min, 70\%-50\% B; 9.01-10.0 $\min 25 \%-5 \%$ B; 10.01-13.0 min 5\% B; 13.01-16.0 min, balance to $70 \%$ B. The detection wavelength was set at $254 \mathrm{~nm}$. A Shim-pack XR-ODS (2.2 $\mu$, $75 \mathrm{~mm} \times 2.0 \mathrm{~mm}$ I.D., Shimadzu) was used to analyze NCHN and its glucuronide. Acetonitrile (A) and Millipore filtered water with $0.2 \%$ formic acid (B) were used as the mobile phase at a flow rate of $0.4 \mathrm{ml} / \mathrm{min}$. The following gradient condition was used: $0-4.0 \mathrm{~min}, 90 \%-25 \% \mathrm{~B}$; 4.01-6.0 $\min 5 \% \mathrm{~B}$; 6.01-8.0 min, balance to $90 \% \mathrm{~B}$. NCHN-O-glucuronide was quantified by liquid chromatography with fluorescence detection with excitation wavelength of $364 \mathrm{~nm}$ and emission wavelength of $450 \mathrm{~nm}$ (Lv et al., 2015).

A LC-MS/MS based method was used to quantify propofol O-glucuronide under the following conditions. Chromatographic separation was performed on an ACCHROM C18HC column (5 $\mu, 150 \mathrm{~mm} \times 2.1 \mathrm{~mm}$, Acchrom Technology Corp., Beijing, China) using an ACQUITYTM UPLC system equipped with a binary pump, a vacuum degasser and an auto-sampler (Waters Corp., Milford, MA, USA). Methanol (A) and Millipore water containing $0.1 \%$ ammonium hydroxide (B) were used as the mobile phase at a flow rate of $0.2 \mathrm{ml} / \mathrm{min}$. The following gradient condition was used: 0-2.0 $\mathrm{min}, 100 \% \mathrm{~B} ; 2.0-6.0 \mathrm{~min}$, $100 \%-10 \% \mathrm{~B}, 6.0-9.0 \mathrm{~min}$, balance to $100 \% \mathrm{~B}$. The mass spectrometry detection was performed on an AB Sciex QTrap® 5500 system with a Turbo VTM source (Framingham, MA, USA). Ionization was operated using an electrospray ionization (ESI) source in the negative ion mode. Optimized instrument settings specific for propofol O-glucuronide and IS (4-methyklumbelliferone-D-glucuronide) were as follows: Curtain Gas was 25 psi, Ion Source Gas 1 was 40 psi, Ion Source Gas 2 was 50 psi, IonSpray Voltage was -4500 V, Turbo 
Heater Temperature was $600{ }^{\circ} \mathrm{C}$. Quantification and confirmation transitions and their optimized values for Entrance Potential (EP), Collision Cell Exit Potential (CXP) were -10 V and $-13 \mathrm{~V}$, respectively. Declustering Potential (DP) for propofol O-glucuronide and IS were -70 psi and -80 psi, respectively. Quantification was performed in multiple reaction monitoring $(\mathrm{MRM})$ mode at $\mathrm{m} / \mathrm{z} \quad 353.2 \rightarrow 177.2$ for propofol O-glucuronide, and $\mathrm{m} / \mathrm{z}$ $351.2 \rightarrow 175.0$ for IS, respectively. The optimized collision energy (CE) was $-35 \mathrm{eV}$ for propofol O-glucuronide, and $-25 \mathrm{eV}$ for IS, respectively. All data acquired were processed using Analyst Ver. 1.5 software (Applied Biosystems, Toronto, Canada).

The calibration curves and linear ranges for quantification of 4-MU and 4-MUG, TFP and its glucuronide, NCHN and its glucuronide, as well as propofol and its glucuronide were constructed by using serial dilutions of the standard solution with appropriate concentration. The calibration curve for each analyte was linear over the tested concentration ranges (from 0.1 to $10 \mu \mathrm{M})$ with good linear regression $\left(\mathrm{R}^{2} \geqslant 0.9998\right)$. The intra-day and inter-day variations in peak area were also evaluated with acceptable relative standard deviation (RSD) values $(<3.8 \%)$. It is also found that all analytes in the sample solutions are stable within $24 \mathrm{~h}$ with acceptable RSD values $(<5.0 \%)$.

\section{Results}

\subsection{Inhibition screening of LCA on the activities of recombinant human UGTs}

To investigate the inhibitory effects of LCA against the catalytic activities of human UGTs, trifluoperazine (TFP) N-glucuronidation (for UGT1A4) and 4-methylumbelliferone (4-MU) O-glucuronidation (for all other UGT isoforms) were used to measure the activities 
of twelve human UGTs with or without LCA. As seen in Fig. 2, upon addition of LCA (10 $\mu \mathrm{M}$, final concentration), the catalytic activities of most human UGTs were strongly inhibited. Specifically, the residual activities of UGT1A1, 1A3, 1A6, 1A7, 1A8, 1A9, 1A10, 2B4, 2B7, 2B15, and 2B17 were 16.7\%, 31.8\%, 12.3\%, 0\%, 75.6\%, 3.2\%, 54.1\%, 61.9\%, 28.2\%, 29.1\%, and $67.8 \%$, respectively, of their null controls. At the same dosage of LCA, the residual activity of UGT1A4-mediated TFP N-glucuronidation was $29.1 \%$. Notably, the catalytic activities of all tested human UGTs could be inhibited by LCA near completely at high concentration. As shown in Fig. 2 upon addition of LCA (100 $\mu$ M, final concentration), the residual activities of UGT1A3 and UGT2B17 were $4.5 \%$, and $6.6 \%$, respectively, while the activities of the other ten human UGTs were totally inhibited.

To further characterize the inhibitory effects of LCA against UGTs activities, dose-dependent inhibition curves were plotted using different LCA concentrations. It can be seen from Fig.3 and Fig.4 that LCA inhibited all the tested UGTs in a concentration-dependent manner. The calculated $\mathrm{IC}_{50}$ values are $0.97 \mu \mathrm{M}, 4.19 \mu \mathrm{M}, 4.26 \mu \mathrm{M}$, $2.52 \mu \mathrm{M}, 0.09 \mu \mathrm{M}, 17.64 \mu \mathrm{M}, 0.37 \mu \mathrm{M}, 15.65 \mu \mathrm{M}, 24.20 \mu \mathrm{M}, 6.37 \mu \mathrm{M}, 14.15 \mu \mathrm{M}$, and 32.05 $\mu \mathrm{M}$ for UGT1A1, 1A3, 1A4, 1A6, 1A7, 1A8, 1A9, 1A10, 2B4, 2B7, 2B15, and 2B17, respectively (Table 1). For comparison, three well-known UGT inhibitors were used as positive controls for inhibition assays. Nilotinib, magnolol, and androsterone are commonly used inhibitors against UGT1A1, UGT1A9, and UGT2B7, respectively (Ai et al., 2014; Zhu et al., 2012; Uchaipichat et al., 2006). The $\mathrm{IC}_{50}$ value of nilotinib against UGT1A1 was determined as $0.10 \mu \mathrm{M}$, while magnolol showed a strong inhibitory effect against UGT1A9 with the $\mathrm{IC}_{50}$ value of $0.08 \mu \mathrm{M}$, and androsterone inhibited UGT2B7 activity with the $\mathrm{IC}_{50}$ 
value of $12.58 \mu \mathrm{M}$. It is evident from these results that LCA is found to be a strong inhibitor against UGT1A1, 1A3, 1A4, 1A6, 1A7, 1A9, and 2B7 ( $\left.\mathrm{IC}_{50}<10 \mu \mathrm{M}\right)$ while serving as a moderate inhibitor against UGT1A8, 1A10, 2B4, 2B15, and 2B17 ( $\mathrm{IC}_{50}$ range from 14 to 32 $\mu \mathrm{M})$. These findings prompted us to further investigate the inhibition kinetic types and the corresponding inhibition parameters of LCA, specifically for UGT1A1, 1A3, 1A4, 1A6, 1A7, 1A9, and 2B7.

\subsection{Kinetic analysis for inhibition of LCA on recombinant UGTs}

To assess the possible mechanisms of LCA inhibition against human UGT isoforms, inhibition kinetic analysis were carried out. As shown by Fig.3, both Lineweaver-Burk and Dixon plots demonstrates that LCA is a competitive inhibitor for UGT1A1-, UGT1A7-, and UGT2B7-mediated 4-MU O-glucuronidation. Second kinetic plots deduced from the Lineweaver-Burk plot slopes were then used to determine the corresponding inhibition parameters. Here, the $K_{i}$ values were determined as $0.78 \mu \mathrm{M}, 0.04 \mu \mathrm{M}$, and $2.12 \mu \mathrm{M}$ for UGT1A1, UGT1A7, and UGT2B7, respectively.

Likewise, Fig.4 shows the noncompetitive inhibition mechanism of LCA against UGT1A3-, UGT1A6-, and UGT1A9-mediated 4-MU O-glucuronidation and against UGT1A4-mediated TFP N-glucuronidation. The $K_{i}$ values of LCA against these four enzymes in Fig.4 were determined as $3.17 \mu \mathrm{M}, 2.32 \mu \mathrm{M}, 2.60 \mu \mathrm{M}$, and $0.43 \mu \mathrm{M}$ for UGT1A3, UGT1A4, UGT1A6, and UGT1A9, respectively. The inhibition types and $K_{i}$ values for these UGTs are listed in Table 1. The inhibition types for UGT1A8, UGT1A10, UGT2B4, UGT2B15, and UGT2B17 were not determined because inhibitory activities of LCA against these UGT isoforms were relatively low. These findings suggest that LCA is a strong and 
non-selective inhibitor of most members from the UGT1A subfamily and of UGT2B7 from the UGT2B subfamily.

\subsection{Kinetic analysis for LCA against NCHN O-glucuronidation in both HLM and UGTIAI}

To explore whether the inhibitory effect of LCA against UGT1A1 is substrate-dependent or enzyme source-dependent, a highly specific UGT1A1 probe substrate, NCHN was used to investigate the inhibitory effect of LCA in both recombinant UGT1A1 and HLM. As shown in Fig.5 and Table 2, LCA displayed potent inhibition against NCHN O-glucuronidation in both UGT1A1 and HLM. The IC $_{50}$ values of LCA against NCHN O-glucuronidation in both UGT1A1 and HLM were $0.13 \mu \mathrm{M}$ and $0.84 \mu \mathrm{M}$, respectively. The $K_{i}$ values for LCA against NCHN O-glucuronidation in both HLM and UGT1A1 were determined as $0.54 \mu \mathrm{M}$ and 0.23 $\mu \mathrm{M}$, respectively. These results together indicated that LCA could potently inhibit UGT1A1 even in complex biological samples such as liver preparations.

\subsection{Kinetic analysis for LCA against propofol O-glucuronidation in both HLM and UGT1A9}

The inhibitory effect of LCA against UGT1A9 was also further investigated in both recombinant human UGT1A9 and HLM by incorporating propofol O-glucuronidation as the probe reaction. As shown in Fig.6 and Table 3, LCA displayed potent inhibition against propofol O-glucuronidation in both UGT1A9 and HLM, with its $\mathrm{IC}_{50}$ values of $0.56 \mu \mathrm{M}$ and $0.26 \mu \mathrm{M}$, respectively. Dixon and Lineweaver-Burk plots revealed that the inhibition type of LCA against UGT1A9 in both recombinant human UGT1A9 and HLM was best fitted as noncompetitive inhibition. The $K_{i}$ values for LCA against propofol O-glucuronidation in both HLM and UGT1A9 were also calculated to be $0.61 \mu \mathrm{M}$ and $0.37 \mu \mathrm{M}$, respectively (Table 3). These findings demonstrate that LCA is a potent inhibitor against UGT1A9 in both 
recombinant UGT1A9 enzyme and human liver preparations.

\subsection{Quantitative prediction of DDI Risks (AUC ratios)}

The magnitudes of this inhibitory interactions of LCA with UGT1A1 and 1A9 were also evaluated by estimating the changes in the AUCs of the substrates that are predominantly metabolized by UGT1A1 or 1A9 after co-administration of two LCA-containing marketed TCM products, HuoxiangZhengqi Tincture and Naolejing Oral Liquid. The AUC ratios were calculated based on several available data, including the hepatic extraction ratio $\left(E_{\mathrm{h}}\right)$, the percentage of hepatic clearance mediated by target UGTs relative to the total clearance of the $\operatorname{substrates}\left(f_{\text {hep }}\right)$, the $K_{i}$ values for LCA against UGT enzymes and the predicted maximum concentration of LCA in human plasma $\left(\mathrm{C}_{\max }\right)$. As shown in Table 4, LCA increased the AUCs of the UGT1A1 substrates by $71-341 \%$ when they were co-administrated with LCA-containing herbal products. Likewise, LCA also increased the AUCs of the UGT1A9 substrates by $64-334 \%$ when they were co-administrated with LCA-containing herbal products.

\section{Discussion}

As an important class of drug-metabolizing enzymes, UGTs participate in metabolic elimination of many endogenous compounds, such as bilirubin and steroids, and account for more than $35 \%$ of phase II drug metabolism (King et al., 2000). However, there is no published report to date regarding the inhibitory effects of LCA against human UGTs. In our study, the inhibitory effects of LCA against human UGTs were investigated for the first time, and the results demonstrate that LCA is a potent and broad-spectrum inhibitor of human 
UGTs. Notably, LCA displayed potent inhibitory effects against several UGTs including UGT1A1, 1A3, 1A4, 1A6, 1A7, $1 \mathrm{~A} 9$ and 2B7, with low $K_{i}$ values ranging from 0.04 to 3.17 $\mu \mathrm{M}$. Although many non-selective UGT inhibitors have been reported (Fang et al., 2013; Jiang et al., 2013; Choi et al., 2014), none of them can inhibit all human UGTs as LCA is able to. It should be noted that LCA $(100 \mu \mathrm{M}$, final concentration) can inhibit the catalytic activities of all tested human UGTs near completely (Fig.2). To the best of our knowledge, LCA is the first reported broad-spectrum inhibitor of human UGTs, and it can be used as a broad-spectrum inhibitor of human UGTs in UGT-related drug metabolism or toxicological studies.

In clinical settings, adverse effects caused by UGT inhibitions have been widely reported in literature. For instance, a strong inhibitory effect of UGT1A1 activity can lead to elevated levels of unconjugated bilirubin in serum following administration of HIV drug, indinavir, and as well as the anticancer agent, sorafenib (Zucker et al., 2001; Meza-Junco et al., 2009). In China, several TCM preparations containing licorice have also been used as adjuvant therapy for the treatment of cancer (Yang et al., 2015). It is also well-established that licorice can be used to reduce mucositis including mouth ulcers (Aly et al., 2005; Agarwal et al., 2009). Moreover, it is well known that UGT1A1 and UGT1A9 are two major metabolizing enzymes responsible for the metabolic elimination of $\mathrm{SN}-38$, the active metabolite of anti-cancer agent irinotecan, which has well-documented adverse events of gastrointestinal perforations (Nagar and Blanchard, 2006; Hecht, 1998). Thus, due to the potent inhibitory effects of LCA against UGT1A1 and UGT1A9 found in this study, the likelihood of the interactions between LCA-containing herbals or TCM preparations and irinotecan is very 
high when they are administered concomitantly. Likewise, LCA has the potential to block the glucuronidation process in human gastrointestinal tract for many UGT substrates, and thus improve the oral bioavailability of many phenolic drugs or natural products. Particularly, for drugs with relatively high therapeutic index that are predominantly metabolized by UGTs, such as the anti-inflammatory agent, daphnetin (Liang et al., 2010), the cholagogue drug, armillarisin A (Sun et al., 2014), the anesthetic agent, propofol (Liang et al., 2011), and the antiretroviral agent, zidovudine (Sun et al., 2015), the co-administration of LCA may extend their half-life and subsequently increase their efficacy in vivo and also potentially reduce the treatment cost.

Unlike the cytochrome P450 enzymes, the UGT enzymes in mammals have significantly varying species differences in both expression and function, while the substrate and inhibitor spectra of the major UGT enzymes are much different from species to species (Higashi et al., 2014; Liang et al., 2011; Guo et al., 2015; Wen et al., 2007). In these cases, we predicted the potential risk of LCA-associated inhibition on human UGTs based on a series of available data (Table 4). In these predictions, the contents of licorice in two marketed TCM products, HuoxiangZhengqi Tincture and Naolejing Oral Liquid, which have been reported were used to calculate the AUCs (Pharmacopoeia of the People`s Republic of China, 2005; Shibata, 2000). The bioavailability of LCA in human has been predicted by a system pharmacology based method (Liu et al., 2013). Based on these data, the maximum plasma concentration of LCA in human was estimated up to 20.8-92.0 $\mu \mathrm{M}$ following administration of the two marketed TCM products. It is evident from this estimated in vivo concentration that the $[\mathrm{I}] / K_{i}$ values are much greater than 1.0 for human UGT1A1, 1A3, 1A4, 1A6, 1A7, 1A9, and 2B7, 
where [I] is the inhibitor level at steady state with the highest clinical dose (Williams et al., 2004). Furthermore, the potential risks of UGT1A1 and 1A9 inhibitions were also predicted based on the inhibitory constant of LCA in HLM. The prediction results show that co-administration of LCA-containing TCM products has the potential to increase the AUCs of substrates that are predominantly metabolized by UGT1A1 or 1A9. It is easily conceivable then that administration of LCA-containing TCM products at high doses may affect the metabolic elimination of bilirubin and consequently lead to the elevated levels of unconjugated bilirubin in human serum as previously discussed. Therefore, for safety considerations, the high dose of LCA-containing herbal preparations should not be used concomitantly with the drugs which are primarily metabolized by the above mentioned seven UGT enzymes (UGT1A1, 1A3, 1A4, 1A6, 1A7, 1A9, and 2B7), especially for those patients with liver injury or hyperbilirubinemia.

Additionally, other factors such as the polymorphism of UGTs might complicate these predictions for HDIs (Burchell et al., 2000). For examples, patients possessing UGT1A1*28 genotype or UGT2B7 mutations (UGT2B7*2, UGT2B7*5, and UGT2B7*71S), the expression and the glucuronidation activity of these enzymes will be relatively lower compared to those of their wild-type counterparts (Zhang et al., 2007; Bjornsson et al., 2003; Coffman et al., 1997). In these genetically polymorphic cases, the patients will have impaired glucuronidation capability for the drugs that are predominantly metabolized by UGT1A1 or UGT2B7. And thus, they are going to be more susceptible to adverse HDIs when LCA-containing herbal preparations are co-administered. This will be particularly true for those drugs with narrow therapeutic index that are substrates for the above mentioned 
polymorphic UGT isoforms. Thus, the concomitant use of licorice is not advised in these cases.

It should also be noted that LCA has been identified as a potent inhibitor against human CYP and P-gp (He et al., 2015; Choi et al., 2014). As the most abundant CYP enzyme in the liver, CYP3A4 is the most important phase I metabolic enzyme that involves biotransformation of up to $50 \%$ of prescribed drugs and numerous xenobiotic (Dorne et al., 2003). P-glycoprotein is an important transporter molecule and is considered to play a key role in absorption, distribution, and elimination of many therapeutic drugs (Balayssac et al., 2005). In this study, our findings demonstrate that LCA is also a potent and broad-spectrum inhibitor of most human UGTs. Therefore, it is very likely that LCA changes the pharmacokinetic profiles of many therapeutic drugs via inhibiting one or more drug metabolizing enzymes, or via inhibiting P-gps, or via inhibiting both drug metabolizing enzymes and P-gps. As such, the strong inhibitory effects of LCA against multiple human metabolizing enzymes and transporters may exacerbate the risk of HDIs in patients following administration of LCA-containing herbal preparations.

In summary, our findings show that LCA is a potent and broad-spectrum inhibitor against human UGT activities. Specifically, LCA displays strong inhibitory effects against UGT1A1, UGT1A3, UGT1A4, UGT1A6, UGT1A7, UGT1A9, and UGT2B7, while shows moderate inhibitory effects against UGT1A8, UGT1A10, UGT2B4, UGT2B15, and UGT2B17. Moreover, our prediction results for in vivo HDIs demonstrate that the interactions are much likely to occur between high dose of LCA and the drugs which are substrates for the above mentioned seven UGTs. The findings from this study are not only useful for guiding 
reasonable applications of LCA and its related medical preparations, but also important in laying out the foundation for further in vivo investigations on the HDI risks caused by LCA-associated inhibition against human UGTs.

\section{Acknowledgments}

This work was supported by the National S\&T Major Projects of China (2012ZX09501001, 2012ZX09506001), the National Basic Research Program of China (2013CB531805), NSF of China $(81473181,81302793,81273590)$, and the Leading Talent Research Fund of Harbin (2011RFXYS079). 


\section{References}

Agarwal, A., Gupta, D., Yadav, G., Goyal, P., Singh, P.K., Singh, U., 2009. An evaluation of the efficacy of licorice gargle for attenuating postoperative sore throat: a prospective, randomized, single-blind study. Anesth. Analg. 109, 77-81.

Ai, L.M., Zhu, L.L., Yang, L., Ge, G.B., Cao, Y.F., Liu, Y., Fang, Z.Z., Zhang, Y.Y., 2014. Selectivity for inhibition of nilotinib on the catalytic activity of human UDP-glucuronosyltransferases. Xenobiotica 44, 320-325.

Aly, A.M., Al-Alousi, L., Salem, H.A., 2005. Licorice: a possible anti-inflammtory and anti-ulcer drug. AAPS PharmSciTech 6, E74-82.

Austin, R.P., Barton P., Cockroft S.L., Wenlock M.C., Riley R.J., 2002, The influence of nonspecific microsomal binding on apparent intrinsic clearance, and its prediction from physicochemical properties. Drug Metab. Dispos. 30; 1497-1503.

Balayssac, D., Authier, N., Cayre, A., Coudore, F., 2005. Does inhibition of P-glycoprotein lead to drug-drug interactions? Toxicol. Lett. 156, 319-329.

Bjornsson, J.D., Callaghan, J.T., Einolf, H.J., Fischer, V., Gan, L., Grimm, S., Kao, J., King, S.P., Miwa, G., Ni, L., Kumar, G., Mcleod, J., Obach, R.S., Roberts, S., Roe, A., Shah, A., Snikeris, F., Sullivan, J.T., Tweedie, D., Vega, J.M., Walsh, J., Wrighton, S.A., 2003. The conduct of in vitro and in vivo drug-drug interaction studies: a Pharmaceutical Research and Manufacturers of America (PhRMA) perspective. Drug Metab. Dispos. 31, 815-832.

Burchell, B., Soars, M., Monaghan, G., Cassidy, A., Smith, D., Ethell, B., 2000. Drug-mediated toxicity caused by genetic deficiency of UDP-glucuronosyltransferases. Toxicol. Lett. 112-113, 333-340. 
Choi, Y.H., Chin, Y.W., Kim, Y.G., 2011. Herb-drug interaction: focus on metabolic enzymes and tranporters. Arch. Pharm. Res. 34, 1843-1863.

Choi, J.S., Choi, J.S., Choi, D.H., 2013. Effects of licochalcone A on the pharmacokinetics of losartan and its active metabolite, EXP-3174, in rats. Pharmazie 68, 882-888.

Choi, E.J., Park, J.B., Yoon, K.D., Bae, S.K., 2014. Evaluation of the in vitro/in vivo potential of five berries (bilberry, blueberry, cranberry, elderberry, and raspberry ketones ) commonly used as herbal supplements to inhibit uridine diphospho-glucuronosyltransferase. Food Chem. Toxicol. 72, 13-19.

Choi, J.S, Choi, J.S., Choi, D.H., 2014. Effects of licochalcone A on the bioavailability and pharmacokinetics of nifedipine in rats: possible role of intestinal CYP3A4 and P-gp inhibition by licochalcone A. Biopharm. Drug Dispos. 35, 382-390.

Coffman, B.L., King, C.D., Rios, G.R., Tephly, T.R., 1998. The glucuronidation of opioids, other xenobiotics, and androgens by human UGT2B7Y(268) and UGT2B7H(268). Drug Metab. Dispos. 26, 73-77.

Dong, R.H., Fang, Z.Z., Zhu, L.L., Liang, S.C., Ge, G.B., Yang, L., Liu, Z.Y., 2012. Investigation of UDP-glucuronosyltransferases (UGTs) inhibitory properties of carvacrol. Phytother. Res. 26, 86-90.

Dorne, J.L.C.M., Walton, K., Renwick, A.G., 2003. Human variability in CYP3A4 metabolism and CYP3A4-related uncertainty factors risk assessment. Food Chem. Toxicol. 41, 201-204.

Fang, Z.Z., Cao, Y.F., Hu, C.M., Hong, M., Sun, X.Y., Ge, G.B., Liu, Y., Zhang, Y.Y., Yang, L., Sun, H.Z., 2013. Structure-inhibition relationship of ginsenosides towards 
UDP-glucuronosyltransferases (UGTs). Toxicol. Appl. Pharmacol. 267, 149-154.

Feldschuh, J., Enson, Y., 1977. Prediction of the normal blood volume. Relation of blood volume to body habitus. Circulation 56, $605-612$.

Friis-Møller, A., Chen, M., Fuursted, K., Christensen, S.B., Kharazmi, A., 2002. In vitro antimycobacterial and antilegionella activity of licochalcone A from Chinese licorice roots. Planta Med. 68, 416-419.

Gregory, P.A., Lewinsky, R.H., Gardner-Stephen, D.A., Mackenzie, P.I., 2004. Regulation of UDP glucuronosyltransferases in the gastrointestinal tract. Toxicol. Appl. Pharm. 199, 354-363.

Guo, B., Fan, X.R., Fang, Z.Z., Cao, Y.F., Hu, C.M., Yang, J.L., Zhang, Y.Y., He, R.R., Zhu, X., Yu, Z.W., Sun, X.Y., Hong, M., Yang, L., 2013. Deglycosylation of liquiritin strongly enhances its inhibitory potential towards UDP-glucuronosyltransferase (UGT) isoforms. Phytother. Res. 27, 1232-1236.

Guo, B., Fang, Z.Z., Yang, L., Xiao, L., Xia, Y.L., Gonzalez, F.J., Zhu, L.L., Cao, Y.F., Ge, G.B., Yang, L., Sun, H.Z., 2015. Tissue and species differences in the glucuronidation of glabridin with UDP-glucuronosyltransferases. Chem Biol Interact. 231, 90-97.

Haraguchi, H., Ishikawa, H., Mizutani, K., Tamura, Y., Kinoshita, T., 1998. Antioxidative and superoxide scavenging activities of retrochalcones in Glycyrrhiza inflata. Bioorgan. Med. Chem. 6, 339-347.

Hatano, T., Kagawa, H., Yasuhara, T., Okuda, T., 1988. Two new flavonoids and other constituents in licorice root: Their relative astringency and radical scavenging effects. Chem. Pharm. Bull. 36, 2090-2097. 
He, Y.H., Fang, Z.Z., Ge, G.B., Jing, P., Jin, H.Z., Zhang, W.D., Yang, L., 2012. The inhibitory effect of 20(S)-protopanaxatriol (ppt) towards UGT1A1 and UGT2B7. Phytother. Res. 27, 628-632.

He, W., Wu, J.J., Ning, J., Hou, J., Xin, H., He, Y.Q., Ge, G.B., Xu, W., 2015. Inhibition of human cytochrone P450 enzymes by licochalcone A, a naturally occurring constituent of licorice. Toxicol. in Vitro 29, 1569-1576.

Hecht, J.R., 1998. Gastrointestinal toxicity or irinotecan. Oncology (Williston Park, N.Y.) 12, $72-78$.

Higashi, E., Ando, A., Iwano, S., Murayama, N., Yamazaki, H., Miyamoto, Y., 2014. Hepatic microsomal UDP-glucuronosyltransferase (UGT) activities in the microminipig. Biopharm Drug Dispos. 35, 313-320.

Jancova, P., Anzenbacher, P., Anzenbacherova, E., 2010. Phase II drug metabolizing enzymes. Biomed Pap. Med. Fac. Univ. Palacky Olomouc Czech Repub. 154, 103-116.

Jiang, H.M., Fang, Z.Z., Cao, Y.F., Hu, C.M., Sun, X.Y., Hong, M., Yang, L., Ge, G.B., Liu, Y., Zhang, Y.Y., Dong, Q., Liu, R.J., 2013. New insights for the risk of bisphenol A: inhibition of UDP-glucuronosyltransferases (UGTs). Chemosphere 93, 1189-1193.

Kiang, T.K., Ensom, M.H., Chang, T.K., 2005. UDP-glucuronosyltransferases and clinical drug-drug interactions. Pharmacol. Therapeut. 106, 97-132.

King, C.D., Rios, G.R., Green, M.D., Tephly, T.R., 2000. UDP-Glucuronosyltransferases. Curr. Drug Metab. 1, 143-161.

Kirby, B.J., Unadkat, J.D., 2010. Impact of ignoring extraction ratio when predicting drug-drug interactions, fraction metabolized, and intestinal first-pass contribution. Drug 
Metab. Dispos.: Biol. Fate Chem. 38, 1926 - 1933.

Kolbe, L., Immeyer, J., Batzer, J., Wensorra, U., tom, Dieck. K., Mundt, C., Woldber, R., Stab, F., Schonrock, U., Ceilley, R.I., Wenck, H., 2006. Anti-inflammatory efficacy of Licochalcone A: correlation of clinical potency and in vitro effects. Arch. Dermatol. Res. 298, 23-30.

Li, L., Hu, H., Xu, S., Zhou, Q., Zeng, S., 2012. Roles of UDP-glucuronosyltransferases in phytochemical metabolism of herbal medicines and the associated herb-drug interactions. Curr. Drug Metab. 13, 615-623.

Liang, S.C., Ge, G.B., Liu, H.Y., Zhang, Y.Y., Wang, L.M., Zhang, J.M., Yin, L., Li, W., Fang, Z.Z., Wu, J.J., Li, G.H., Yang, L., 2010. Identification and characterization of human UDP-glucuronosyltransferases responsible for the in vitro glucuronidation of daphnetin. Drug Metab. Dispos. 38, 973-980.

Liang, S.C., Ge, G.B., Liu, H.Y., Shang, H.T., Wei, H., Fang, Z.Z., Zhu, L.L., Mao, Y.X., Yang., L., 2011. Determination of propofol UDP-glucuronosyltransferase (UGT) activities in hepatic microsomes from different species by UFLC-ESI-MS. J. Pham. Biomed. Anal. 54, 236-241.

Lin, A.L., Shangari, N., Chan, T.S., Remirez, D., O`Brien, P.J., 2006. Herbal monoterpene alcohols inhibit propofol metabolism and prolong anesthesia time. Life Sci. 79, 21-29.

Liu, C., Cao, Y.F., Fang, Z.Z., Zhang, Y.Y., Hu, C.M., Sun, X.Y., Huang, T., Zeng, J., Fan, X.R., Hong, M., 2012. Strong inhibition of deoxyschizandrin and schisantherin A toward UDP-glucuronosyltransferase (UGT) 1A3 indicating UGT inhibition-based herb-drug interaction. Fitoterapia 83, 1415-1419. 
Liu, J.L., Pei, M.J., Zheng, C.L., Li, Y., Wang, Y.H., Lu, A.P., Yang, L., 2013. A systems-pharmacology analysis of herb medicines used in health improvement treatment: predicting potential new drugs and targets. Evid. Based Complement. Alternat. Med. 2013, 938764.

Liu, J.M., Wang, Q.L., Yao, T.W., Yang, B.C., 2014. Research on distribution and dosage of liquorice. Chinese Arch. Tradit. Chin. Med. 32, 3021-3024.

Lu, H., Fang, Z.Z., Cao, Y.F., Hu, C.M., Hong, M., Sun, X.Y., Li, H., Liu, Y., Fu, X.G., Sun, H.Z., 2013. Isoliquiritigenin showed strong inhibitory effects towards multiple UDP-glucuronosyltransferase (UGT) isoform-catalyzed 4-methylumbelliferone (4-MU) glucuronidation. Fitoterapia 84, 208-212.

Lv, X., Ge, G.B., Feng, L., Troberg, J., Hu, L.H., Hou, J., Cheng, H.L., Wang, P., Liu, Z.M., Finel, M., Cui, J.N., 2015. An optimized ratiometric fluorescent probe for sensing human UDP-glucuronosyltransferase 1A1 and its biological applications. Biosens. Bioelectron. 72, 261-267.

Meza-Junco, J., Chu, Q.S., Christensen, O., Rajagopalan, P., Das, S., Stefanyschyn, R., Sawyer, M.B., 2009. UGT1A1 polymorphism and hyperbilirubinemia in a patient who received sorafenib. Cancer Chemoth. Pharm. 65, 1-4.

Miners, J.O., Bowalgaha, K., Elliot, D.J., Baranczewski, P., Knighter, K.M., 2011. Characterization of niflumic acid as a selective inhibitor of human liver microsomal UDP-glucuronosyltransferase 1A9: application to the reaction phenotyping of acetaminophen glucuronidation. Drug Metab. Dispos. 39, 644-652.

Na, D.H., Ji, H.Y., Park, E.J., Kim, M.S., Liu, K.H., Lee, H.S., 2011. Evaluation of 
metabolism-mediated herb-drug interactions. Arch. Pharm. Res. 34, 1829-1842.

Nagar, S., Blanchard, R.L., 2006. Pharmacogentics of uridine diphosphoglucuronosyltransferase (UGT) 1A family members and its role in patient response to irinotecan. Drug Metab. Rev. 38, 393-400.

Pharmacopoeia of the People`s Republic of China, 2005. Chemical Industry Press.

Park, M.R., Kim, S.G., Cho, I.A., Oh, D., Kang, K.R., Lee, S.Y., Moon, S.M., Cho, S.S., Yoon, G., Kim, C.S., Oh, J.S., You, J.S., Kim, D.K., Seo, Y.S., Im, H.J., Kim, J.S., 2015. Licochalcone-A induces intrinsic and extrinsic apoptosis via ERK1/2 and p38 phosphorylation-mediated TRAIL expression in head and neck squamous carcinoma FaDu cells. Food Chem. Toxicol. 77, 34-43.

Qiao, X., Ji, S., Yu, S.W., Lin, X.H., Jin, H.W., Duan, Y.K., Zhang, L.R., Guo, D.A., Ye, M., 2014. Identification of key licorice constituents which interact with Cytochrome P450: Evaluation by LC/MS/MS cocktail assay and metabolic profiling. APPS J. 16, 101-113.

Ritter, J.K., 2000. Roles of glucuronidation and UDP-glucuronosyltransferases in xenobiotic bioctivation reactions. Chem. Biol. Interact. 129, 171-193.

Rowland, A., Knights, K.M., Mackenzie, P.I., Miners, J.O., 2008. The "albumin effect" and drug glucuronidation: bovine serum albumin and fatty acid-free human serum albumin enhance the glucuronidation of UDP-glururonosyltransferase (UGT) 1A9 substrates but not UGT1A1 and UGT1A6 activities. Drug Metab. Dispos. 36, 1056-1062.

Rowland, A., Miners, J.O., Mackenzie, P.I., 2013. The UDP-glucuronosyltransferases: their role in drug etabolism and detoxification. Int. J. Biochem. cell Biol. 45, 1121-1132.

Shibata, S., 2000. A drug over the millennia: pharmacognosy, chemistry, and pharmacology 
of licorice. Yakugaku Zasshi 120, 849-862.

Song, J.H., Cui, L., An, L.B., Li, W.T., Fang, Z.Z., Zhang, Y.Y., Dong, P.P., Wu, X., Wang, L.X., Gonzalez, F.J., Sun, X.Y., Zhao, D.W., 2015. Inhibition of UDP-glucuronosyltransferases (UGTs) activity by constituents of Schisandra chinensis. Phytother. Res. 29, 1658-1664.

Sun, D.X., Zhu, L.L., Xiao, L., Xia, Y.L., Ge, G.B., Cao, Y.F., Wu, Y., Yin, J., Yang, L., 2014. In vitro glucuronidation of Armillarisin A: UDP-glucuronosyltransferase 1A9 acts as a major contributor and significant species differences. Xenobiotica 44, 988-995.

Sun, H., Zhang, T.P., Wu, Z.F., Wu, B.J., 2015. Warfain is an effective modifier of multiple UDP-Glucuronosyltransferase enzymes: Evaluation of its potential to alter the pharmacokinetics of zidovudine. J. Pharm. Sci. 104, 244-256.

Uchaipichat, V., Mackenzie, P.I., Guo, X.H., Gardner-Stephen, D., Galetin, A., Houston, J.B., Miners, J.O., 2004. Human UDP-glucuronosyltransferases: isoform selectivity and kinetics of 4-methylumbelliferone and 1-naphthol glucuronidation, effects of organic solvents, and inhibition by diclofenac and probenecid. Drug Metab. Dispos. 32, 423-432.

Uchaipichat, V., Mackenzie, P.I., Elliot, D.J., Miners, J.O., 2006. Selectivity of substrate (trifluoperazine) and inhibitor (amitriptyline, androsterone, canrenoic acid, hecogenin, phenylbutazone, quinidine, quinine, and sulfinpyrazone) "probes" for human UDP-glucuronosyltransferases. Drug Metab. Dispos. 34, 449-456.

Wang, X.X., Lv, X., Li, S.Y., Hou, J., Ning, J., Wang, J.Y., Cao, Y.F., Ge, G.B., Guo, B., Yang, L., 2015. Identification and characterization of naturally occurring inhibitors against UDP-glucuronosyltransferase 1A1 in Fructus Psoraleae (Bu-gu-zhi). Toxicol Appl 
Pharmacol 289, 70-78.

Wen, Z.M., Martin, D.E., Bullock, P., Lee, K.H., Smith, P.C., 2007. Glucuronidation of anti-HIV drug candidate bevirimat: identification of human UDP-glucuronosyltransferases and species differences. Drug Metab. Dispos. 35, 440-448.

Williams, J.A., Hyland, R., Jones, B.C., Smith, D.A., Hurst, S., Goosen, T.C., Peterkin, V., Koup, J.R., Ball, S.E., 2004. Drug-drug interactions for UDP-glucuronosyltransferase substrates: a pharmacokinetic explanation for typically observed low exposure (AUCi/AUC) ratios. Drug Metab. Dispos. 32, 1201-1208.

Wu, B., Kulkarni, K., Basu, S., Zhang, S., Hu, M., 2011. First-pass metabolism via UDP-glucuronosyltransferase: a barrier to oral bioavailability of phenolics. J. Pharm. Sci. $100,3655-3681$.

Xia, Y.L., Ge, G.B., Wang, P., Liang, S.C., He, Y.Q., Ning, J., Qian, X.K., Li, Y., Yang, L., 2015. Structural modifications at the C-4 position strongly affect the glucuronidation of 6,7-dihydroxycoumarins. Drug Metab. Dispos. 43, 553-560.

Xiao, X.Y., Hao, M., Yang, X.Y., Ba, Q., Li, M., Ni, S.J., Wang, L.S., Du, X., 2011. Licochalcone A inhibits growth of gastric cancer cells by arresting cell cycle progression and inducing apoptosis. Cancer Lett. 302, 69-75.

Yang, X.B., Wu, W.Y., Long, S.Q., Deng, H., Pan, Z.Q., He, W.F., Zhou, Y.S., Liao, G.Y., Li, Q.P., Xiao, S.J., Cai, J.Z., 2015. Fuzheng Kang`ai decoction combined with gefitinib in advanced non-small cell lung cancer patients with epidermal growth factor receptor mutations: study protocol for a randomized controlled trial. Trials 16, 146-152.

Yo, Y.T., Shieh, G.S., Hsu, K.F., Wu, C.L., Shiau, A.L., 2009. Licorice and licochalcone-A 
induce autophagy in LNCaP prostate cancer cells by suppression of Bcl-2 expression and the mTOR pathway. J. Arg. Food Chem. 57, 8266-8273.

Zhang, D.L., Zhang, D.X., Cui, D., Gambardella, J., Ma, L., Barros, A., Wang, L.F., Fu, Y.L., Rahematpura, S., Nielsen, J., Donegan, M., Zhang, H.J., Humphreys, W.G., 2007. Characterization of the UDP Glucuronosyltransferase activity of human liver microsomes genotyped for the UGT1A1*28 polymorphism. Drug Metab. Dispos. 35, 2270-2280.

Zhang, L., Lin, G., Zuo, Z., 2007. Involvement of UDP-glucuronosyltransferases in the extensive liver and intestinal first-pass metabolism of flavonoid baicalein. Pharm. Res. 24, 81-89.

Zhang, Q.Y., Ye, M., 2009. Chemical analysis of Chinese herbal medicine Gan-Cao (licorice). J. Chromatogr. A 1216, 1954-1969.

Zheng, Y.F., Bae, S.H., Choi, E.J., Park, J.B., Kim, S.O., Jang, M.J., Park, G.H., Shin, W.G., Oh, E., Bae, S.K., 2014. Evaluation of the in vitro/in vivo drug interaction potential of BST204, a purified dry extract of ginseng, and its four bioactive ginsenosides through cytochrome P450 inhibition/induction and UDP-glucuronosyltransferase inhibition. Food Chem. Toxicol. 68, 117-127.

Zhu, L.L., Ge, G.B., Liu, Y., He, G.Y., Liang, S.C., Fang, Z.Z., Dong, P.P., Cao, Y.F., Yang, L., 2012. Potent and selective inhibition of magnolol on catalytic activities of UGT1A7 and 1A9. Xenobiotica 42, 1001-1008.

Ziegler, H.L., Hansen, H.S., Staerk, D., Christensen, S.B., Hagerstrand, H., Jaroszewski, J.W., 2004. The antiparasitic compound licochalcone a is a potent echinocytogenic agent that modifies the erythrocyte membrane in the concentration range where antiplasmodial 
activity is observed. Antimicrob. Agents Chemother. 48, 4067-4071.

Zucker, S.D., Qin, X., Rouster, S.D., Yu, F., Green, R.M., Keshavan, P., Feinberg, J., Sherman, K.E., 2001. Mechanism of indinavir-induced hyperbilirubinemia. Proc. Natl. Acad. Sci. U. S. A. $98,12671-12676$. 


\section{Figure Legends}

Figure 1. The chemical structure of LicochalconeA (LCA)

Figure 2. The inhibitory effects of LCA $(1 \mu \mathrm{M}, 10 \mu \mathrm{M}$, and $100 \mu \mathrm{M})$ on the catalytic activities of human UGT isoforms. The data are presented as the means \pm the S.D.s of triplicate determinations.

Figure 3. Inhibition curves and Lineweaver-Burk plots showing competitive inhibitions by LCA against UGT1A1 (A), UGT1A7 (B), and UGT2B7 (C). Left: the dose-dependent inhibition curves; Right: the Lineweaver-Burk plots showing competitive inhibitions. All data represent the means of triplicate determinations.

Figure 4. Inhibition curves and Lineweaver-Burk plots showing noncompetitive inhibitions by LCA against UGT1A3 (A), UGT1A4 (B), UGT1A6 (C) and UGT1A9 (D). Left: the dose-dependent inhibition curves; Right: the Lineweaver-Burk plots showing noncompetitive inhibitions. All data represent the means of triplicate determinations.

Figure 5. Inhibition curves and Lineweaver-Burk plots of LCA against UGT1A1-mediated NCHN-O-glucuronidation in both HLM (A) and recombinant human UGT1A1 (B). Left: the dose-dependent inhibition curves; Right: the Lineweaver-Burk plots showing noncompetitive inhibitions. All data represent the means of triplicate determinations.

Figure 6. Inhibition curves and Lineweaver-Burk plots of LCA against UGT1A9-mediated propofol-O-glucuronidation in both HLM (A) and recombinant humanUGT1A9 (B). Left: the dose-dependent inhibition curves; Right: the Lineweaver-Burk plots showing noncompetitive inhibitions. All data represent the means of triplicate determinations. 
Table 1. Inhibition constants of LCA towards twelve human UGTs.

\begin{tabular}{|c|c|c|c|c|c|}
\hline Enzyme & Substrate & $\mathrm{IC}_{50}(\boldsymbol{\mu M})$ & $K_{\mathbf{i}}(\boldsymbol{\mu M})$ & Type of inhibition & Goodness of Fit $\left(\mathbf{R}^{2}\right)$ \\
\hline UGT1A1 & 4-MU & $0.97 \pm 0.08$ & 0.78 & Competitive & 0.99 \\
\hline UGT1A3 & 4-MU & $4.19 \pm 0.35$ & 3.17 & Noncompetitive & 0.99 \\
\hline UGT1A4 & TFP & $4.26 \pm 0.32$ & 2.32 & Noncompetitive & 0.99 \\
\hline UGT1A6 & 4-MU & $2.52 \pm 0.26$ & 2.6 & Noncompetitive & 0.99 \\
\hline UGT1A7 & 4-MU & $0.09 \pm 0.02$ & 0.04 & Competitive & 0.99 \\
\hline UGT1A8 & 4-MU & $17.64 \pm 2.92$ & -- & -- & -- \\
\hline UGT1A9 & 4-MU & $0.37 \pm 0.03$ & 0.43 & Noncompetitive & 1.00 \\
\hline UGT1A10 & 4-MU & $15.65 \pm 1.79$ & -- & -- & -- \\
\hline UGT2B4 & 4-MU & $24.20 \pm 3.73$ & -- & -- & -- \\
\hline UGT2B7 & 4-MU & $6.37 \pm 0.84$ & 2.12 & Competitive & 0.99 \\
\hline UGT2B15 & 4-MU & $14.15 \pm 2.39$ & -- & -- & -- \\
\hline UGT2B17 & 4-MU & $32.05 \pm 4.25$ & -- & -- & -- \\
\hline
\end{tabular}


Table 2. Inhibition constants of LCA against NCHN-O-glucuronidation in both HLM and recombinant human UGT1A1.

\begin{tabular}{ccclc}
\hline Enzyme source & $\mathbf{I C}_{\mathbf{5 0}}(\boldsymbol{\mu M})$ & $\boldsymbol{K}_{\mathbf{i}}(\boldsymbol{\mu M})$ & Type of inhibition & Goodness of Fit $\left(\mathbf{R}^{\mathbf{2}}\right)$ \\
\hline HLM & $0.84 \pm 0.09$ & 0.54 & Noncompetitive & 0.97 \\
UGT1A1 & $0.13 \pm 0.02$ & 0.23 & Noncompetitive & 1.00 \\
\hline
\end{tabular}

Table 3. Inhibition constants of LCA against propofol-O-glucuronidation in both HLM and recombinant human UGT1A9.

\begin{tabular}{ccccc}
\hline Enzyme source & $\mathbf{I C}_{\mathbf{5 0}}(\boldsymbol{\mu M})$ & $\boldsymbol{K}_{\mathbf{i}}(\boldsymbol{\mu M})$ & Type of inhibition & Goodness of Fit $\left(\mathbf{R}^{\mathbf{2}}\right)$ \\
\hline HLM & $0.26 \pm 0.03$ & 0.61 & Noncompetitive & 0.99 \\
UGT1A9 & $0.56 \pm 0.07$ & 0.37 & Noncompetitive & 0.96 \\
\hline
\end{tabular}


Table 4. Prediction of the potential herb-drug interaction risks of LCA in vivo based on the AUC ratios.

\begin{tabular}{ccccccc}
\hline Substrate & $E_{\mathrm{h}}{ }^{\mathrm{a}}$ & $f_{\text {hep }}{ }^{\mathrm{b}}$ & $K_{\mathrm{i}}(\mu \mathrm{M})$ & $I(\mu \mathrm{M})^{\mathrm{c}}$ & AUC ratios ${ }^{\mathrm{d}}$ & AUC increase (\%) \\
\hline UGT1A1 Substrate & & & 0.54 & & $1.71-4.41$ & $71-341 \%$ \\
& $0.4-0.9$ & 0.8 & & $5.82-25.8$ & & \\
UGT1A9 Substrate & & & 0.61 & & $1.64-4.34$ & $64-334 \%$ \\
\hline
\end{tabular}

a. $E_{\mathrm{h}}$ is the hepatic extraction ratio ranging from 0.4 to 0.9 for UGT substrates.

b. The $f_{\text {hep }}$ was set to the mean value of each UGT substrates.

c. The maxmum concentration of inhibitor $(I)$ in human plama was caluculated as $f_{\mathrm{u}} \times \mathrm{C}_{\max }$. The crude extract of Liquorice is a major material for producing several famous marketed TCM products (e.g., HuoxiangZhengqi Tincture and Naolejing Oral Liquid). The daily dose of these two marketed TCM products contained the crude extract from 20-35.4 $\mathrm{g}$ of Liquorice. LCA accounts for approximately 0.4-1\% (w/w) of liquorice (Shibata, 2000). An estimated oral bioavailability of LCA in human has been reported as $45.75 \%$ (Liu et al., 2013). The average volume of human blood is approximately 5.2 L (Feldschuh and Enson, 1977). On the basis of the above data, the $\mathrm{C}_{\max }$ of LCA in humans was estimated up to 20.8-92.0 $\mu \mathrm{M}$. In addition, $f_{\mathrm{u}}$ is the unbound fraction of LCA in plasma, which was predicted as 0.28 by using the following equation (Austin et al., 2002).

$$
f_{u}=\frac{1}{[P] \cdot 10^{0.56 \cdot \log (\mathrm{P} / \mathrm{D})-1.41}+1}
$$

Here $[P]$ is the mean value of human plasma protein concentration $(70 \mathrm{mg} / \mathrm{ml})$.

d. Prediction methods as described in the Materials and Methods. 
<smiles>C=CC(C)(C)c1cc(/C=C/C(=O)c2ccc(O)cc2)c(OC)cc1O</smiles>

Fig. 1. The chemical structure of Licochalcone A (LCA)

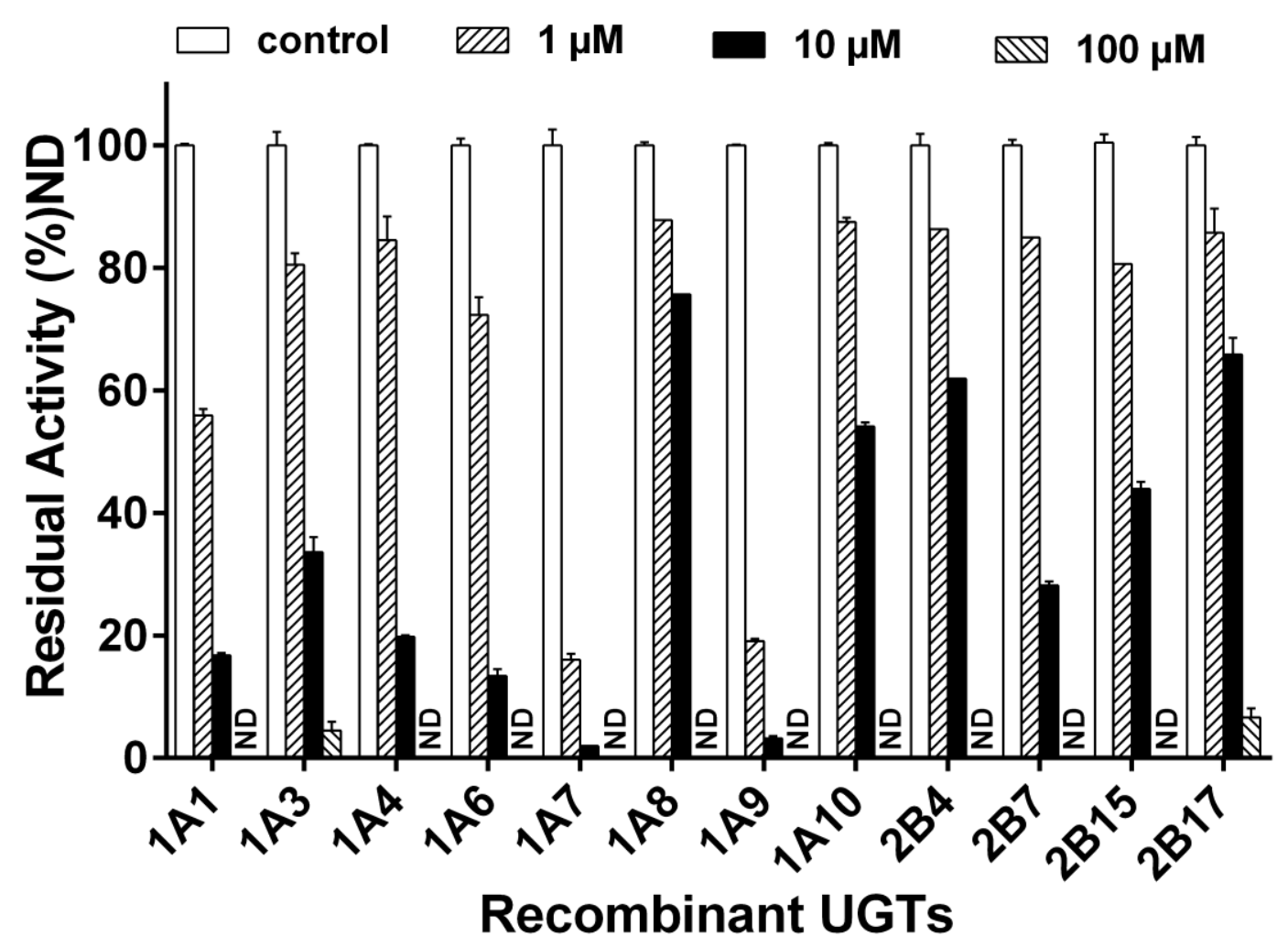

Fig. 2.The inhibitory effects of LCA $(1 \mu \mathrm{M}, 10 \mu \mathrm{M}$, and $100 \mu \mathrm{M})$ on the catalytic activities of human UGT isoforms. The data are presented as mean \pm S.D. of triplicate determinations. 

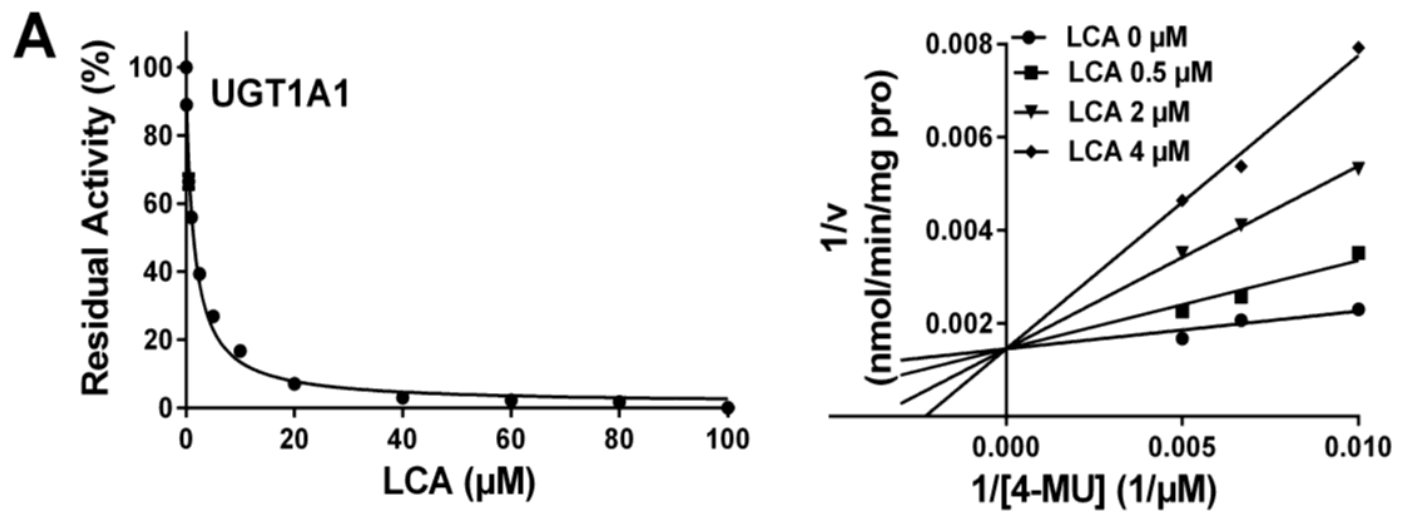

B
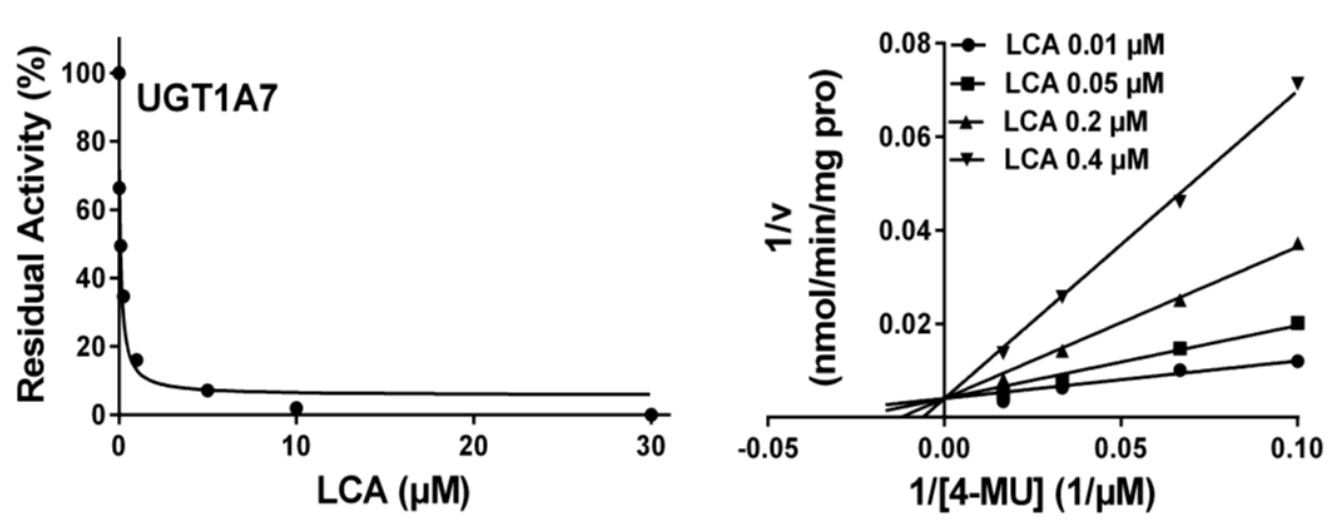

C
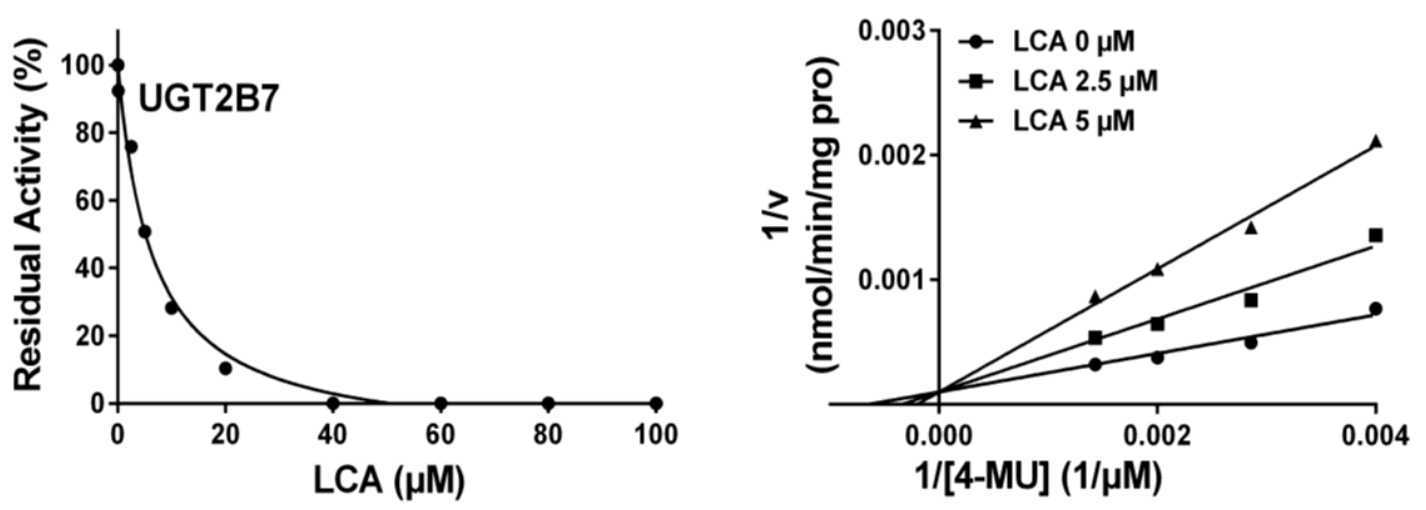

Fig. 3. Inhibition curves and Lineweaver-Burk plots showing competitive inhibitions by LCA against UGT1A1 (A), UGT1A7 (B), and UGT2B7 (C). Left: the dose-dependent inhibition curves; Right: the Lineweaver-Burk plots showing competitive inhibitions. All data represent the means of triplicate determinations. 

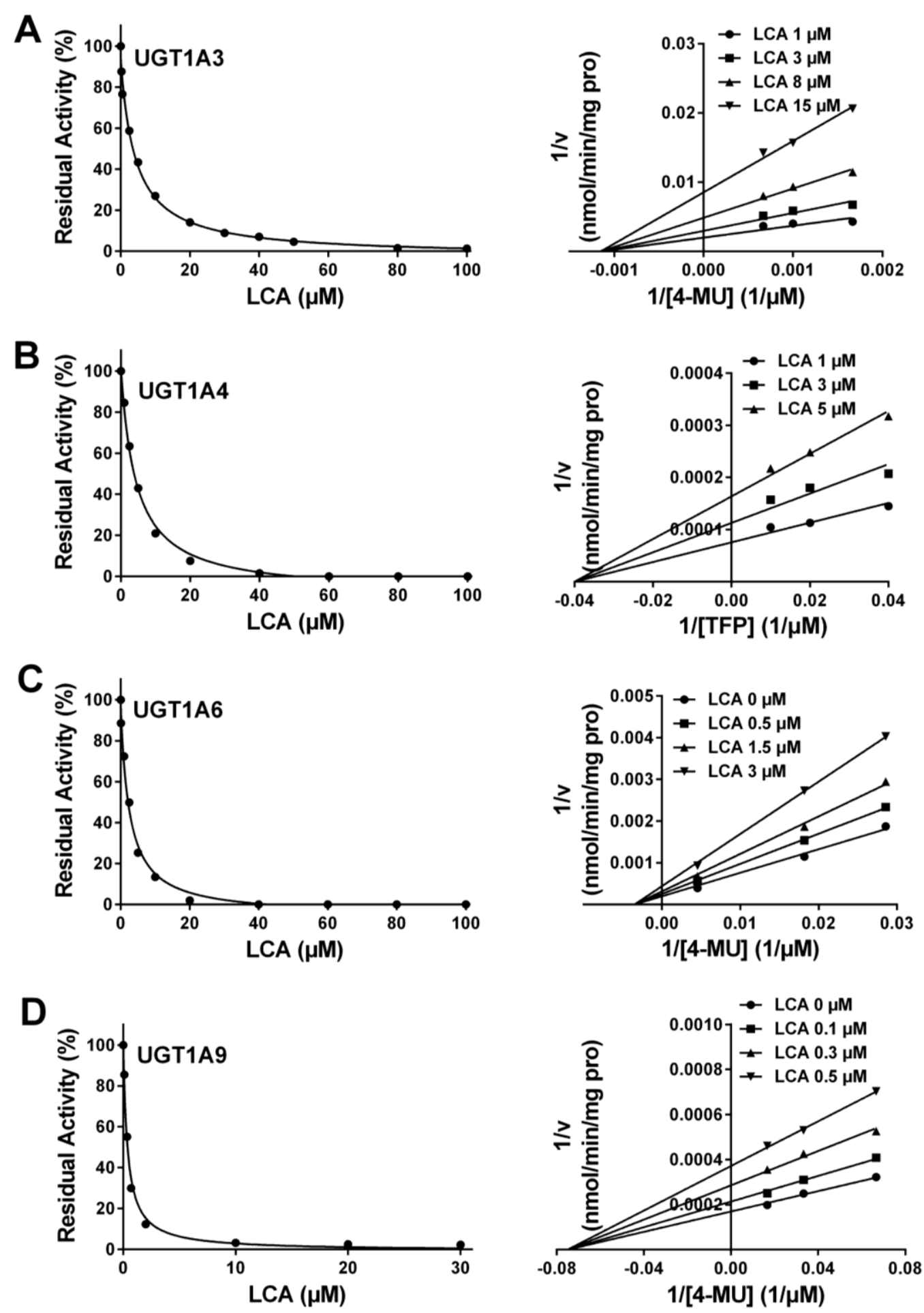

Fig. 4. Inhibition curves and Lineweaver-Burk plots showing noncompetitive inhibitions by LCA against UGT1A3 (A), UGT1A4 (B), UGT1A6 (C) and UGT1A9 (D). Left: the dose-dependent inhibition curves; Right: the Lineweaver-Burk plots showing noncompetitive inhibitions. All data represent the means of triplicate determinations. 
A

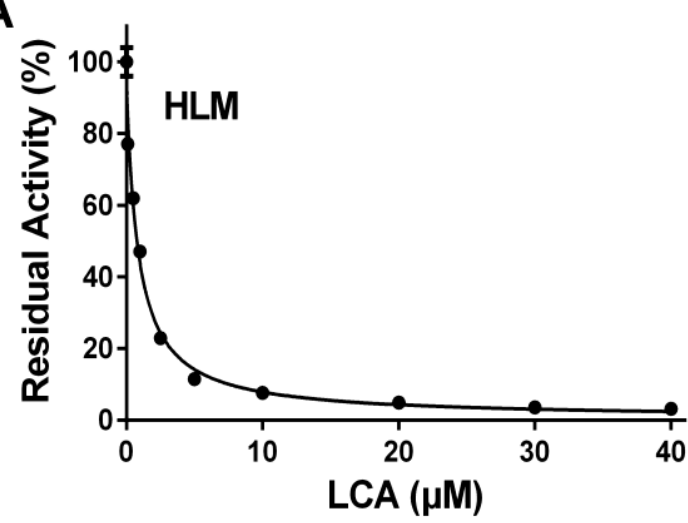

B

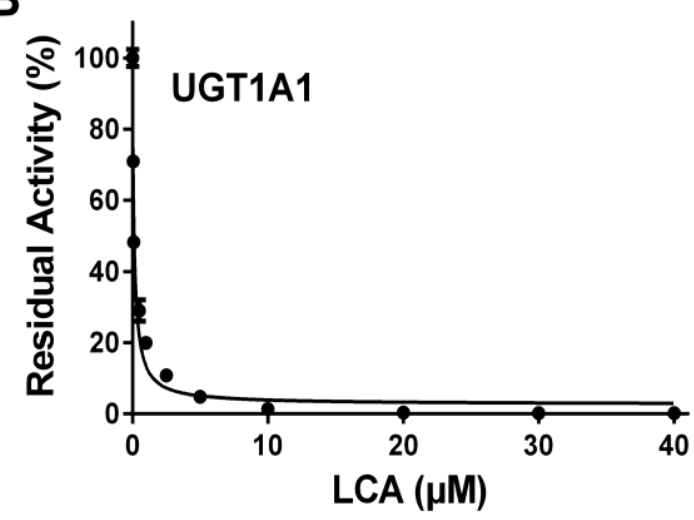

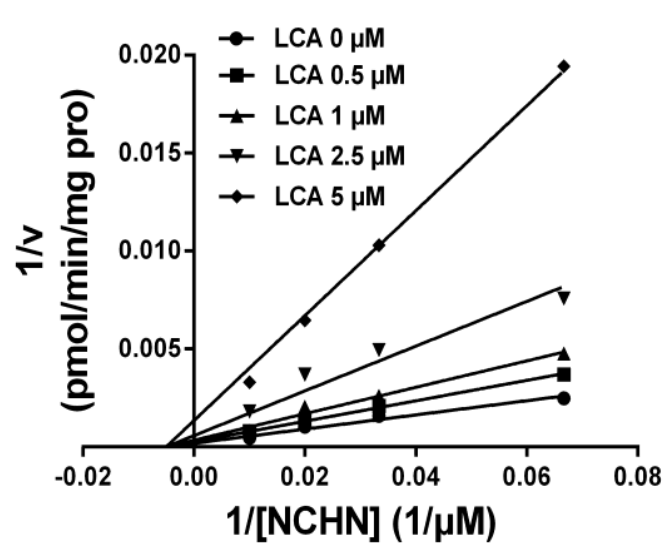

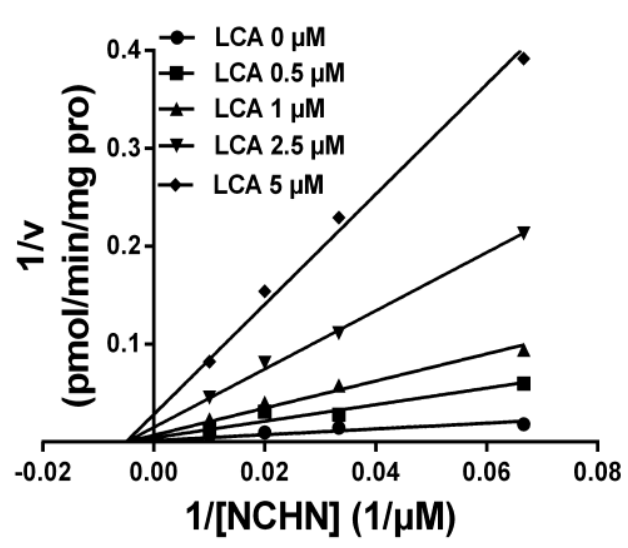

Fig. 5. Inhibition curves and Lineweaver-Burk plots of LCA against UGT1A1-mediated NCHN-O-glucuronidation in both HLM (A) and recombinant human UGT1A1 (B). Left: the dose-dependent inhibition curves; Right: the Lineweaver-Burk plots showing noncompetitive inhibitions. All data represent the means of triplicate determinations. 

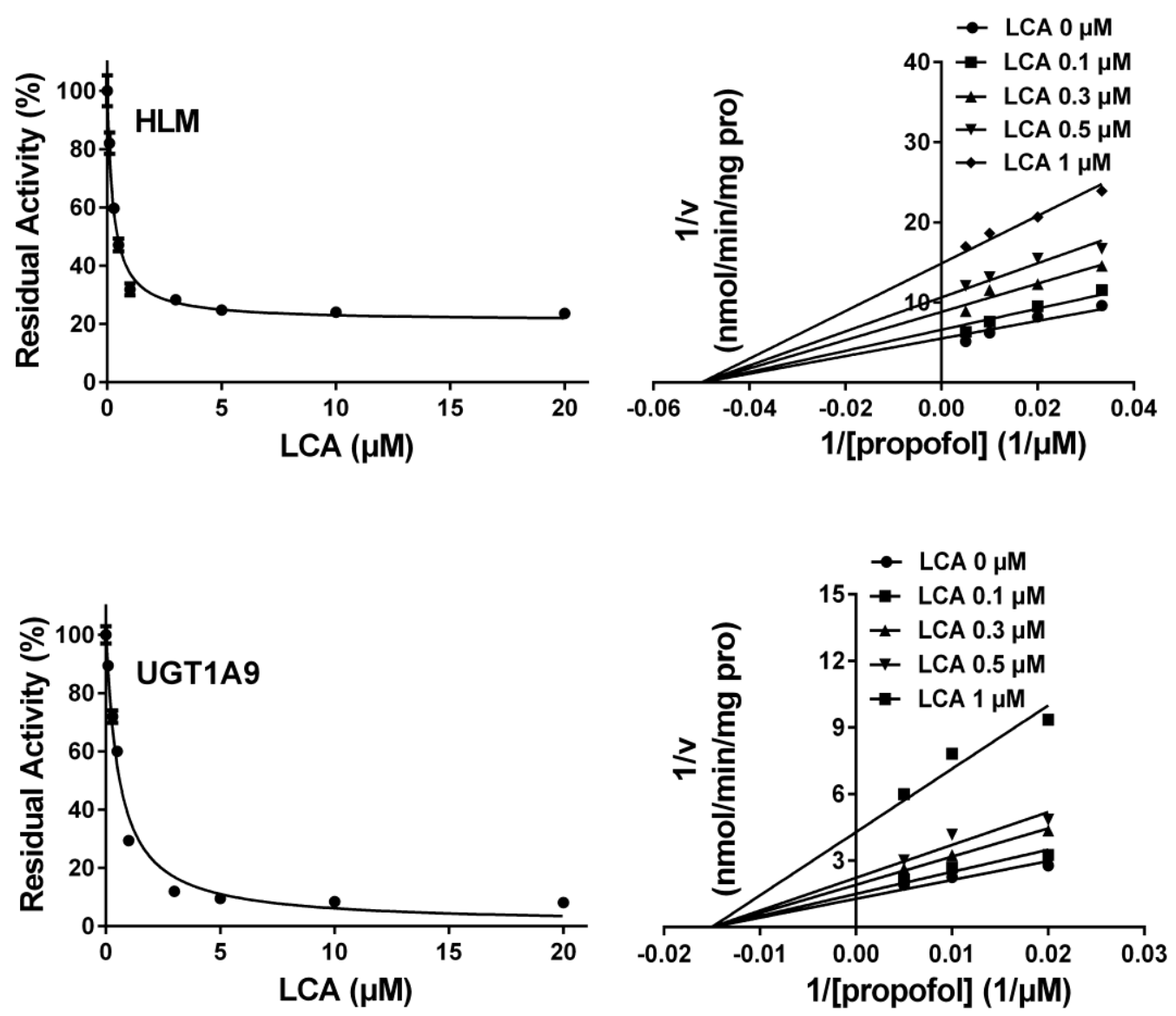

Fig.6. Inhibition curves and Lineweaver-Burk plots of LCA against UGT1A9-mediated propofol-O-glucuronidation in both HLM (A) and recombinant human UGT1A9 (B). Left: the dose-dependent inhibition curves; Right: the Lineweaver-Burk plots showing noncompetitive inhibitions. All data represent the means of triplicate determinations. 